\title{
Uso de imunoglobulinas na terapêutica da púrpura trombocitopênica imune
}

\author{
Use of immunoglobulins in immune thrombocytopenic purpura therapy
}

\author{
Ercínia Gomes Duailibe Barros ${ }^{1}$, Jéssica Gomes Duailibe Barros ${ }^{1}$, Lise Feitosa Novais \\ Miranda1, Gissela Santos Lindoso¹, Lilalea Gonçalves França ${ }^{1}$
}

Resumo: A trombocitopenia é um achado comum na prática clínica e a Púrpura Trombocitopênica Imune (PTI) é uma de suas várias causas, antigamente considerada idiopática. Caracteriza-se por doença autoimune com queda da quantidade absoluta de plaquetas e aumento do risco de sangramento. Para fins diagnósticos, divide-se em recentemente diagnosticada, persistente e crônica. Entre os 30 e 60 anos é mais prevalente em mulheres, tornando-se equivalente em ambos os sexos após essa idade e sendo mais autolimitada na infância. Entre os possíveis sintomas, podem ocorrer petéquias, equimoses, sangramento de mucosas, e outros, sendo que nem todos os pacientes apresentam sintomas. A primeira-linha terapêutica da PTI é composta por corticosteroides, imunoglobulina intravenosa (IgIV) e imunoglobulina anti-D. Ambas as imunoglobulinas levam tempos semelhantes para uma resposta inicial. Apesar dos estudos acerca dos mecanismos de ação da IglV e seu potencial de supressão de auto-anticorpos, a literatura não é clara acerca de sua dose ótima, tempo de infusão e desconsidera seu alto custo neste tratamento. A anti-D pode ser alternativamente utilizada em paciente $R h(D)$-positivos e com baço intacto. Ensaios clínicos adicionais são indispensáveis para otimizar o custo-efetividade na terapêutica da $\mathrm{PTI}$, além de reduzir sua toxicidade.

Palavras-chave: Púrpura. Trombocitopenia. Imunoglobulina.

\begin{abstract}
Thrombocytopenia is a common finding in clinical practice and Immune Thrombocytopenic Purpura (ITP) is one of its several causes, previously called idiopathic. It is characterized by an autoimmune disease with a decrease in the absolute platelets count and increased risk of bleeding. For diagnostic purposes, it is divided into recently diagnosed, persistent and chronic. Between 30 and 60 years, it is more prevalent in women, becoming equivalent in both sexes after this age and being more self-limiting in childhood. Possible symptoms include petechiae, ecchymosis, mucosal bleeding, and others, and not all patients have symptoms. The first-line therapy for ITP is composed of corticosteroids, intravenous immunoglobulin (IVIg) and anti-D immunoglobulin. Both immunoglobulins take similar times for an initial response. Despite studies about the mechanisms of action of IVIg and its potential for suppression of autoantibodies, the literature is not clear about its optimal dose, infusion time, and disregard its high cost in this treatment. Anti-D can alternatively be used in Rh(D)-positive patients with intact spleen. More clinical trials are indispensable to optimize cost-effectiveness in ITP therapy, in addition to reducing its toxicity.
\end{abstract}

Keywords: Purpura. Thrombocytopenia. Immunoglobulin.

\footnotetext{
${ }^{1}$ Universidade Ceuma, São Luís, MA, Brasil.

* Autor correspondente - Rua Josué Montello nำ1, Renascença II - São Luís-MA CEP 65.075120. Telefone:981021569. E-mail: lilafranca@gmail.com
} 


\section{Introdução}

A produção das plaquetas é realizada pela medula óssea, sendo regulada principalmente pelo hormônio trombopoietina que estimula a sua síntese $e$ a diferenciação de megacariócitos. Seu principal papel está no controle de sangramentos e hemorragias, pois estas circulam constantemente no sangue em seu estado inativo, e auxiliam na nutrição endotelial e preservação da integridade do vaso. A trombocitopenia, definida como a contagem de plaquetas menor de $100 \times 10 \%$, é comum na prática clínica, mas apresenta dificuldades no diagnóstico e no seu manejo ${ }^{1}$.

A hemostasia envolve um balanço delicado entre fatores anticoagulantes e procoagulantes. Os principais componentes da hemostasia são as plaquetas, fatores de coagulação, inibidores da coagulação, fribinólise e vasos sanguíneos. $\mathrm{Na}$ trombocitopenia grave, este balanço pode estar descompensado, induzindo a sangramento de caráter mucocutâneo ${ }^{1}$.

As plaquetas, em especial, podem apresentar deficiências qualitativas e/ou quantitativas. Uma das causas de trombocitopenia quantitativa é a púrpura trombocitopênica imunológica (PTI), doença adquirida com destruição autoimune das plaquetas e associada à supressão da produção plaquetária pelos megacariócitos da medula óssea, com ou sem complicação hemorrágica ${ }^{1,2}$.

Para os pacientes com PTI, a escolha do tratamento inclui: esteroides, imunoglobulina intravenosa (lgIV), imunoglobulina anti-D, esplenectomia, rituximab, agentes estimuladores da trombopoiese e a terapia "watch and wait" ${ }^{3}$. Atualmente, as terapias de primeira linha padrão na contenção da trombocitopenia grave $e$ sangramento incluem corticosteroides, imunoglobulina intravenosa (lgIV, $1-2 \mathrm{mg} / \mathrm{kg}$ ) e imunoglobulina anti-D (anti-D), que são efetivos para mais de $80 \%$ dos pacientes ${ }^{1,4,5}$.

Este estudo tem como objetivo principal discorrer, por meio de uma revisão da literatura, sobre os diversos conhecimentos acerca do uso de imunoglobulinas na terapêutica da Púrpura Trombocitopênica Imune (PTI). A definição, patogênese, classificações, epidemiologia e características clínicas dessa doença serão brevemente apresentados.

\section{Métodos}

\section{Tipo de Estudo}

Trata-se de um estudo desenvolvido por revisão de literatura, de caráter qualitativo e de natureza analítica e exploratória.

\section{Métodos e instrumentos de coleta de dados}

O estudo foi realizado por meio da busca de artigos publicados em inglês referentes ao Uso de Imunoglobulinas na Terapêutica da Púrpura Trombocitopênica Imune. Foram utilizados os seguintes bancos de dados: SciELO (Scientific Electronic Library Online), PubMed.

\section{Universo e amostra}

Foram consultadas 2 bases de dados, obtendo uma amostra de 13 (treze) artigos sobre a temática desta 
revisão (entre artigos e dissertações).

\section{Critérios de inclusão e exclusão}

Os critérios de inclusão
utilizados foram artigos que
atendessem à temática proposta; no
idioma inglês; com texto completo
disponível; e num espaço temporal
de até quatorze anos devido o tema
ser pouco abordado pela
comunidade científica. Os critérios
de exclusão foram artigos que se
repetiam nas bases de dados e que
fugiam à temática

\section{Análise de dados}

Os trabalhos encontrados por meio da pesquisa passaram por uma leitura detalhada do resumo e do corpo, onde foram identificados pontos importantes e separados para uma leitura mais aprofunda e guiada para selecionar o conteúdo.

\section{Resultados}

A Púrpura Trombocitopênica Imune é uma doença mediada por auto-anticorpos caracterizada pela queda da contagem de plaquetas (< $100 \times 10^{9}$ ) e um risco aumentado de sangramento ${ }^{1,4}$. Sua via é a perda da tolerância do sistema imune aos auto-antígenos localizados na superfície das plaquetas e megacariócitos. Essencialmente, a PTI resulta de anticorpos antiplaquetários produzidos por células $B$, tendo frequentemente como alvo glicoproteínas primárias das plaquetas, como a GP Ila/IIIB. Apesar do entendimento de alguns mecanismos, a fisiopatologia da PTI é complexa e muitas interações permanecem indeterminadas ${ }^{1}$.

Essa entidade patológica é dividida em duas categorias maiores, cuja proporção de indivíduos é variável e não tão bem definida: PTI primária (a maioria dos casos na maior parte dos estudos), na qual outras condições associadas à trombocitopenia estão ausentes; e PTI secundária, que pode ser devida a numerosos agentes infecciosos, doenças linfoproliferativas e autoimunes de base, além de drogas, como a quinina ${ }^{5}$.

Outras classificações também foram desenvolvidas para facilitar as decisões no manejo, como segue: recentemente diagnosticado (diagnóstico há 3 meses), persistente (3-12 meses) e crônico (> 12 meses). A PTI grave é definida por sangramento à apresentação suficiente para demandar tratamento, ou novos sintomas hemorrágicos requerendo intervenções terapêuticas adicionais com um agente elevador de plaquetas diferente ou uma dose aumentada do agente atual ${ }^{1,5}$.

Acreditou-se por mais de meio século, e até pouco tempo, que a PTI era uma doença que acometia primariamente mulheres, sobretudo entre suas terceira e quarta décadas de vida. Entretanto, os estudos epidemiológicos mais recentes têm demonstrado que a incidência mais elevada de PTI idade-específica ocorre durante a velhice. Este fenômeno reflete potencialmente 0 desenvolvimento de desregulação imune com uma consequência do envelhecimento. A preponderância das mulheres na PTI durante a fase adulta ocorre até os 60 anos, período após 0 qual a incidência global cresce em ambos os sexos, e a proporção entre mulheres e homens afetados é praticamente similar. Assim, embora a trombocitopenia no idoso possa refletir mielodisplasia, a $\mathrm{PTI}$ é mais comum do que se acreditava previamente 5,6 . 
Na infância, a PTI apresenta um pico entre os 5 e 6 anos, e se comporta diferentemente de como no adulto. Nas crianças, geralmente se segue a uma aparente infecção viral e tende a ser autolimitada, com aproximadamente $80 \%$ dos casos resolvidos espontaneamente dentro dos 6 meses. Já nos adultos, a doença frequentemente se torna crônica ${ }^{5,7}$.

Acerca das manifestações clínicas, a PTI tem sido reconhecida como uma síndrome diversa com uma diversidadede sinais e sintomas, tais como petéquias, equimose (púrpura seca), sangramento de mucosas (púrpura úmida) e outras manifestações hemorrágicas, que podem incluir sangramento menstrual intenso, sangramento oral e espitaxe. O sangramento está geralmente, embora não inteiramente, ligado à contagem de plaquetas. Mais da metade dos pacientes com uma contagem plaquetária entre 30 e 50 x $10^{9} /$ L não apresentam sintomas ${ }^{1,5}$. da PTI

Imunoglobulinas na terapêutica

Até hoje, a maioria das medidas de tratamento não foi capaz de alterar o curso natural da PTI. Não raras vezes, os efeitos adversos podem sobrepor os benefícios do tratamento. Para os pacientes que requerem tratamento, a primeira meta é remover o risco de sangramento fatal e aumentar a contagem de plaquetas para níveis seguros o mais rápido possível. Enquanto a IglV e a anti-RhD, entre os diferentes tratamentos para PTI, compartilham o tempo mais curto para a resposta inicial (1-3 dias) e para o pico da resposta (3-7 dias), a segunda pode induzir a hemólise intravascular grave ${ }^{3}$.

Imunoglobulina Intravenosa
As primeiras fontes mostrando que a IgIV tinha 0 potencial imunomodulador e supressor de doenças autoimunes foram obtidas em crianças com hipogamaglobulinemia. De forma interessante, pacientes com baixos níveis de $\lg G$ no soro frequentemente produzem autoanticorpos especifícos para vários auto-antígenos, incluindo anticorpos específicos das plaquetas. Além da indicação da IgIV na PTI, seu uso terapêutico também é atualmente licenciado para 0 tratamento da doença de Kawasaki, síndrome de Guillain-Barré, entre outras ${ }^{8}$.

A imunoglobulina intravenosa (IgIV) é usada como uma terapia de substituição em indivíduos imunodeficientes, uma vez que estes não são incapazes de estruturar suas próprias respostas imunes efetivas. Menos intuitivamente, a IgIV também pode ser usada para suprimir as respostas imunes patológicas que ocorrem em pacientes com autoimunidade. $O$ maior componente das preparações de IgIV é a fração lgG do soro, que é acumulada de vários milhares de doadores. Anticorpos lgG são importantes para proteger 0 hospedeiro de infecções microbianas, mas seus autoanticorpos são os maiores colaboradores para a patologia de diversas doenças autoimunes ${ }^{9}$.

Estruturalmente falando, a IgIV pode ser vislumbrada, para fins de entendimento, possuindo quatro componentes isolados: (1) ações mediadas pelas regiões variáveis $F(a b ') 2$, (2) ações da região $F c$ ao alcance de receptores Fc (FcR), (3) ações mediadas pela ligação do complemento dentro do fragmento Fc e (4) outras substâncias imunomodulatórias, que não 0 anticorpo, encontradas nas 
preparações da lg. Deve-se lembrar que nem todos os potenciais mecanismos de ação cabem perfeitamente nesses grupos e que vários podem ocorrer concomitantemente ${ }^{8}$. O mecanismo de ação mais aceito da IgIV na PTI é o bloqueio de receptores Fcy nos macrófagos, prevenindo assim a destruição pelo sistema reticuloendotelial das plaquetas sensibilizadas pelo IgG. Várias estratégias moleculares para desenvolver uma imunoglobulina IV aprimorada estão em andamento, bem como o uso da lg subcutânea na doença autoimune vem sendo explorada ${ }^{10}$.

Embora estudos anteriores sobre a imunoglobulina intravenosa tenham se dedicado a compreender seus mecanismos e contribuíram bastante na sua aplicação como uma importante terapia para pacientes com PTI, estes mesmos estudos desprezaram um fator chave: o pesado fardo financeiro por sua dose convencional imposto aos pacientes. Como uma droga de alto custo, o qual excede o custo do romiplostim (um agonista da trombopoetina, útil em elevar a contagem das plaquetas), a IgIV faz-se frequentemente inacessível para muitos destes pacientes ${ }^{3}$.

A dose da IgIV para a PTI variou nas literaturas de 0,4 a 2 $\mathrm{g} / \mathrm{kg} / \mathrm{dia}$, com infusões variando de 1 a 5 dias. Ensaios mostraram que a dose de $1 \mathrm{~g} / \mathrm{kg} /$ dia por 1-2 dias parece ser a dose ótima (quando administrada como agente isolado). Geralmente, IgIV é administrada por várias horas. Doses mais altas não melhoraram a resposta plaquetária e doses menores ou infusão mais lenta podem ser utilizadas, no intuito de reduzir a toxicidade, uma vez que a eficácia já se mostrou comprovadamente equivalente ${ }^{3,11}$.
Numerosos ensaios controlados mostraram taxas de resposta inicial comparáveis àquelas de corticosteroides, mas com um tempo mais curto de resposta 7 .

Entre os efeitos colaterais da IgIV estão cefaleias, de moderadas a graves, neutropenia transitória, insuficiência renal, meningite asséptica, trombose, rubor, febre, calafrios, fadiga, náuseas, diarreia, alterações da pressão arterial e taquicardia. As preparações de IgIV podem conter pequenas quantidades de $\lg \mathrm{A}, 0$ que ocasionalmente causa reações anafiláticas em pacientes com deficiência de $\lg A$. Nestes casos, deve-se optar por IgIV com depleção de $\lg A^{2,7,11}$. Aparentemente em alguns indivíduos os corticosteroides podem aumentar a resposta da IgIV. Além disso, o seu uso concomitante pode reduzir as reações à infusão e prevenir meningite asséptica? ${ }^{7}$. Imunoglobulina Anti-D

A imunoglobulina Anti-Rh(D) ou anti-D é uma alternativa à IgIV em pacientes $R h(D)$-positivos e não esplenectomizados ${ }^{5,7}$. Anti-Rh(D) se liga a hemácias Rh-positivas, levando-as a serem removidas no sistema reticuloendotelial e bloqueando a remoção de plaquetas marcadas pelo anticorpo. Desse modo, hemácias são sacrificadas para salvar plaquetas, mas porque a quantidade daquelas é maior do que a dessas, fazendo com que os benefícios geralmente superem os riscos. ${ }^{6}$ Deve ser, nesse sentido, evitada naqueles indivíduos com anemia hemolítica autoimune, para evitar exacerbação da hemólise ${ }^{7}$.

Anti-Rh(D) foi originalmente preparada para prevenir a doença hemolítica em recém-nascidos. Há mais de 30 anos tem sido utilizada para tratar pacientes com trombocitopenia imune. Em 1995, a 
imunoglobulina anti-D foi licenciada nos Estados Unidos pela Food and Drugs Association (FDA) para ser utilizada tratamento de PTI aguda em crianças que não passaram por esplenectomia e RhD-positivas, além de crianças e adultos com PTI secundária a HIV ${ }^{12,13}$.

O regime de dosagem aprovada para a terapia anti-D nos Estados Unidos é $50 \mu \mathrm{\mu g} / \mathrm{kg}$. Entretanto, permanecem algumas questões, como se doses mais altas podem ser mais seguras e mais eficazes. Estudos anteriores mostraram que uma resposta plaquetária máxima a $50 \mu \mathrm{g} / \mathrm{kg} / \mathrm{d}$ ocorreu $72 \mathrm{~h}$ após a infusão intravenosa. Outro estudo realizado com indivíduos com contagem de plaquetas $<30 \times 10 \%$ L resultou em maiores aumentos médios no número de plaquetas nos dias 1 e 7 , quando comparados à menor dose, sem uma queda significativamente grande da hemoglobina. Entre os efeitos colaterais mais comuns estão anemia hemolítica (toxicidade doselimitada), calafrios/febre e, raramente, hemólise intravascular, coagulação intravascular disseminada, falência renal e morte.

\section{Considerações finais}

\footnotetext{
Apesar dos grandes avanços no conhecimento acerca dos mecanismos fisiopatológicos e manejo da PTI, a literatura ainda é bastante escassa de ensaios clínicos randomizados e em diretrizes que sustentem a escolha de condutas em detrimento da experiência profissional. A persistência da ideia de que a PTI ainda se trata de um "diagnóstico de exceção" alerta para a necessidade de maior aprofundamento e definição de seus fatores de risco, bem como de
}

preditores de resposta à terapia e marcadores diagnósticos. Quanto ao tratamento, apesar do êxito no uso das imunoglobulinas IgIV e anti-D, não se pode ignorar sua difícil acessibilidade por fatores socioeconômicos, bem como a demanda de estudos de coorte prospectivos e multicêntricos que avaliem as possibilidades de outras linhas terapêuticas e otimizem 0 custo-efetividade no cuidado dessa população, além de reduzir a toxicidade. Apenas dessa forma, será possível, direcionar os planos de tratamento para cada caso. 


\section{Referências}

1. Bolton-Maggs $P$. The management of immune thrombocytopenic purpura. Current Paediatrics. 2002;12(4):298-303.

2. Neunert CE. Current management of immune thrombocytopenia. Hematology Am Soc Hematol Educ Program. 2013;2013(1):276-82.

3. Zhou Z, Qiao Z, Li H, Luo N, Zhang X, Xue $F$, et al. Different dosages of intravenous immunoglobulin (IVIg) in treating immune thrombocytopenia with long-term follow-up of three years: Results of a prospective study including 167 cases. Autoimmunity. 2016;49(1):50-7.

4. Zeller MP, Heddle NM, Kelton JG, Hamilton K, Wang G, Sholapur N, et al. Effect of a thrombopoietin receptor agonist on use of intravenous immune globulin in patients with immune thrombocytopenia. Transfusion. 2016;56(1):73-9.

5. McCRAE K. Immune thrombocytopenia: no longer 'idiopathic'. Cleve Clin J Med. 2011;78(6):358 .

6. Cines DB, Bussel JB. How I treat idiopathic thrombocytopenic purpura (ITP). Blood. 2005;106(7):2244-51.

7. Provan D, Stasi R, Newland AC, Blanchette VS, Bolton-Maggs P, Bussel $\mathrm{JB}$, et al. International consensus report on the investigation and management of primary immune thrombocytopenia. Blood. 2010;115(2):168-86.

8. Jolles S, Sewell W, Misbah S. Clinical uses of intravenous immunoglobulin. Clin Exp Immunol. 2005;142(1):1-11.

9. Schwab I, Nimmerjahn F. Intravenous immunoglobulin therapy: how does $\lg G$ modulate the immune system? Nat Rev Immunol. 2013;13(3):176.

10.Kovaleva L, Apte S, Damodar S, Ramanan V, Loriya S, Navarro-Puerto J, et al. Safety and efficacy of a $10 \%$ intravenous immunoglobulin preparation in patients with immune thrombocytopenic purpura: results of two international, multicenter studies. Immunotherapy. 2016;8(12):1371-81.

11.Spahr JE, Rodgers GM. Treatment of immune-mediated thrombocytopenia purpura with concurrent intravenous immunoglobulin and platelet transfusion: a retrospective review of 40 patients. Am J Hematol. 2008;83(2):122-5.

12. Longhurst $\mathrm{H}$, O'grady $\mathrm{C}$, Evans G, De Lord C, Hughes A, Cavenagh J, et al. Anti-D immunoglobulin treatment for thrombocytopenia associated with primary antibody deficiency. J Clin Pathol. 2002;55(1):64-6.

13.Li J, E van der Wal D, Zhu L, Vadasz B, K Simpson E, Li C, et al. Fc-independent phagocytosis: implications for IVIG and other therapies in immune-mediated thrombocytopenia Cardiovasc Hematol Disord Drug Targets. 2013;13(1):50-8. 